\title{
The permeation of gastric epithelial cells by leucocytes
}

\author{
D. D. GIBBS \\ From the Department of Medicine, The London Hospital
}

EDITORIAL SYNOPSIS The cytological observations reported here favour the occurrence of epithelial infiltration by leucocytes rather than a simple transmigration of white cells through the epithelium.

In descriptions of the histological features of simple and atrophic gastritis attention has been directed to polymorphonuclear leucocytes which have often appeared to be migrating from the lamina propria through the surface epithelium. Thus, Magnus (1946) commented on 'the presence between the (epithelial) cells of large numbers of vacuoles which contain polymorphonuclear leucocytes. These cells are present in large numbers in the overlying mucus'. Motteram (1951), in a biopsy study of chronic gastritis and gastric atrophy, noted that 'neutrophil polymorphs were numerous close to the surface and around the pits. They can be seen surrounded by a small clear space in various stages of migration through the limiting membrane'. It might be deduced from these and other descriptions (Konjetzny, 1930; Palmer, 1954) that polymorphonuclear leucocytes reach the lumen of the stomach by migration between surface epithelial cells. The precise location of leucocytes within the epithelial pallisade is difficult to define in histological preparations. As part of a recent study (Gibbs, 1962) observations were made on exfoliated cells recovered in saline washings from the stomach, and the cytological features were then compared with the histological appearances in gastric mucosal suction biopsies taken from the same patients. In a number of patients with superficial or atrophic gastritis, isolated epithelial cells or small cell groups showing intracytoplasmic inclusions of leucocytes were recovered in washings. This phenomenon in gastric epithelium has received little attention. In addition, cells which contain leucocytic fragments have usually been misinterpreted as histiocytes by cytologists when examining exfoliated material from the stomach. The permeation of gastric epithelial cells by leucocytes sometimes seen in gastritis is now recorded and illustrated.

\section{MATERIAL AMD METHODS}

Examinations by gastric exfoliative cytology were made on 128 patients in whom there was some suspicion of gastric or oesophageal carcinoma (Gibbs, 1962). The observations reported now were derived from eight patients and in these gastric carcinoma was excluded as far as possible by full investigation. Changes of superficial or atrophic gastritis were present in biopsy specimens taken from the stomach of each patient either by suction biopsy or at laparotomy. Two of the patients were achlorhydric after augmented histamine tests, and in one of these there was clinically manifest pernicious anaemia at the time of examination. The stomachs of the other patients were found to secrete acid, which was present either in resting juice or in response to histamine.

Cells exfoliated into the stomach were collected by saline washings. After an overnight fast a rubber tube was swallowed by each patient. Resting juice was withdrawn from the stomach and lavage was then performed using 200 to $400 \mathrm{ml}$. of physiological saline. The fluid was centrifuged with a minimum of delay (3,000 r.p.m. for five minutes) and the deposits were used to make eight smears from each patient. Smears were immediately fixed, before they had dried, in spirit (industrial 74 O.P.) or in $95 \%$ alcohol. They were stained by Papanicolaou's method (Papanicolaou, 1942). After dehydration and clearing they were mounted in Canada balsam.

Mucosal biopsy specimens were obtained from six patients using Crosby's capsule (Crosby and Kugler, 1957). Before each biopsy was taken the position of the capsule was determined by fluoroscopy, and a site was chosen either along the greater curve of the body of the stomach or in the antrum. In two patients full-thickness biopsies were taken at the time of laparotomy. Biopsy specimens were fixed in formalin and sections stained by haematoxylin and eosin.

\section{OBSERVATIONS}

Biopsies were taken from the stomachs of eight patients because of the distinctive appearances of a proportion of the epithelial cells obtained by gastric lavage in these patients. Examples of isolated cells and small sheets and clusters of epithelial cells that were exfoliated are illustrated in Figures 1 to 4 . Varying degrees of degenerative change were 


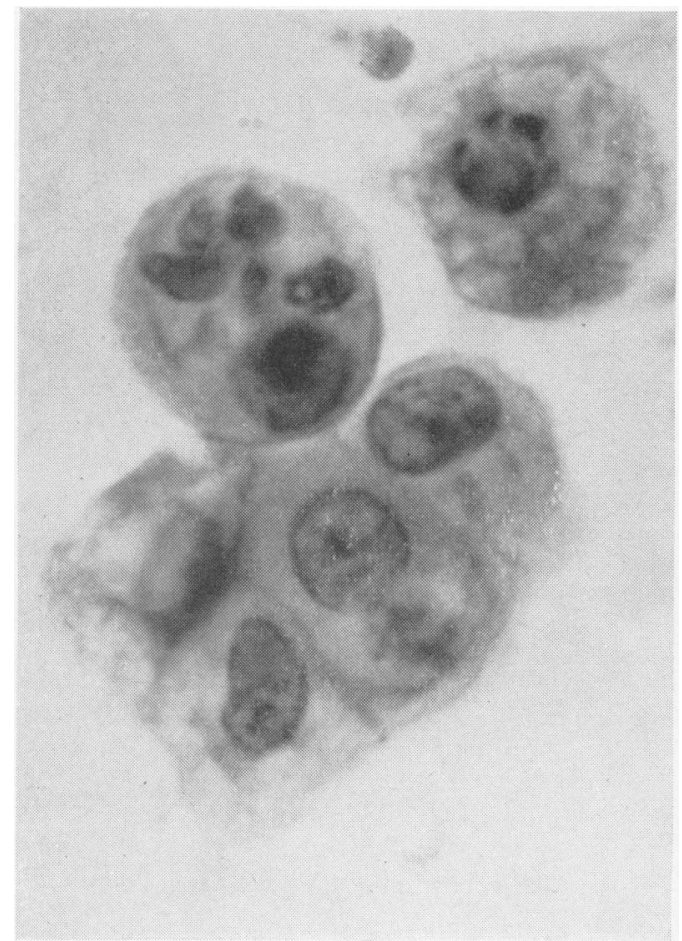

FIG. 1

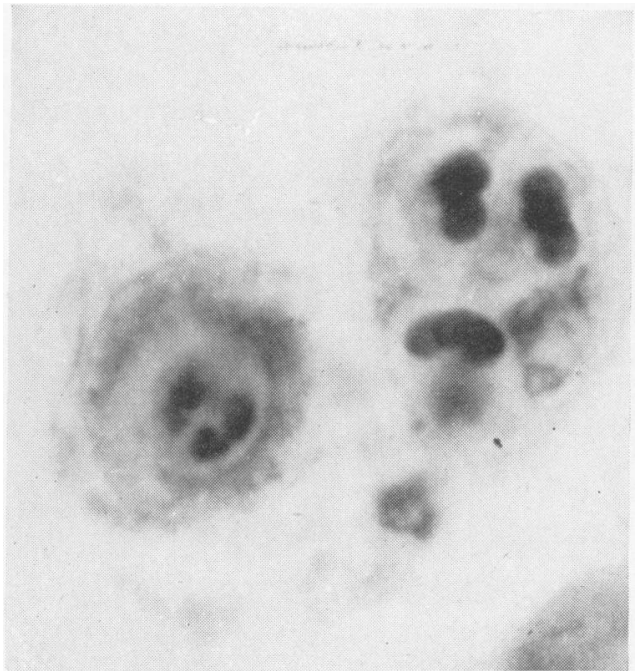

FIG. 2

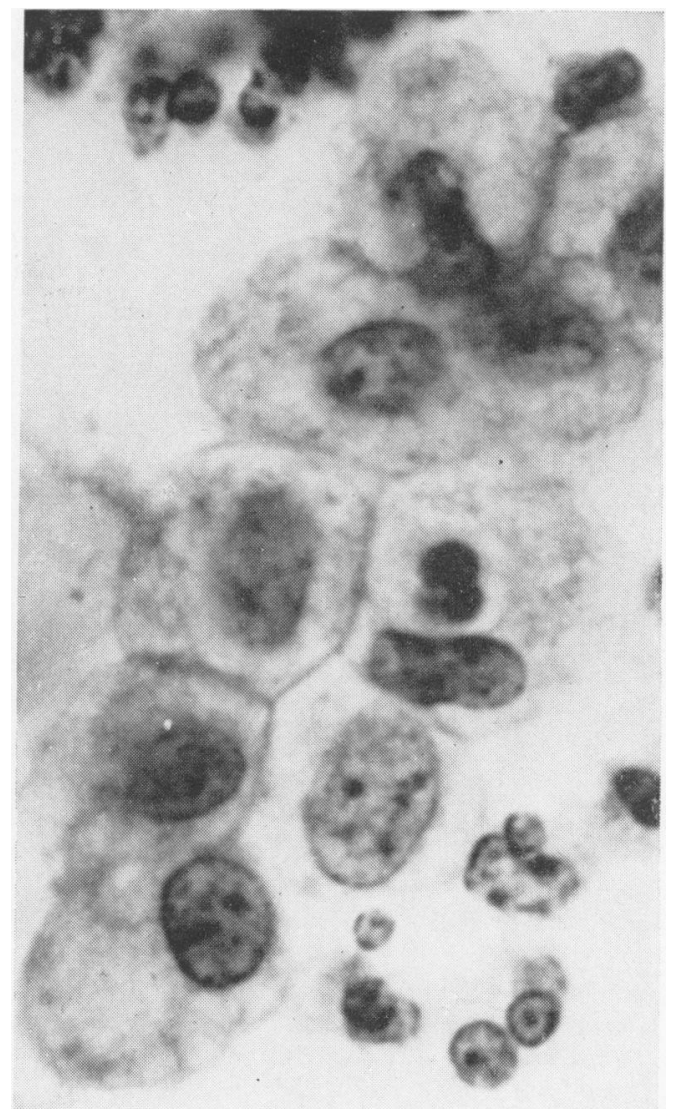

FIG. 3

FIG. 1. A group of gastric epithelial cells recovered by lavage. In one of these leucocytic fragments are present. The cells illustrate several stages of degenerative change. Papanicolaou $\times 840$. Enlarged $\times 4$.

FIG. 2. Two cells which show features of advanced degeneration but in which nuclei of leucocytes are clearly visible. Such cells have often been designated phagocytes or histiocytes by cytologists but are probably epithelial cells invaded by leucocytes before exfoliation. Papanicolaou $\times 840$. Enlarged $\times 4$.

FIG. 3. A small sheet of gastric epithelium recovered from a patient in whom gastric biopsy showed the presence of superficial gastritis. In the cytoplasm of one cell is the nucleus of a leucocyte partially surrounded by a clear halo. Papanicolaou $\times 840$. Enlarged $\times 4$. 


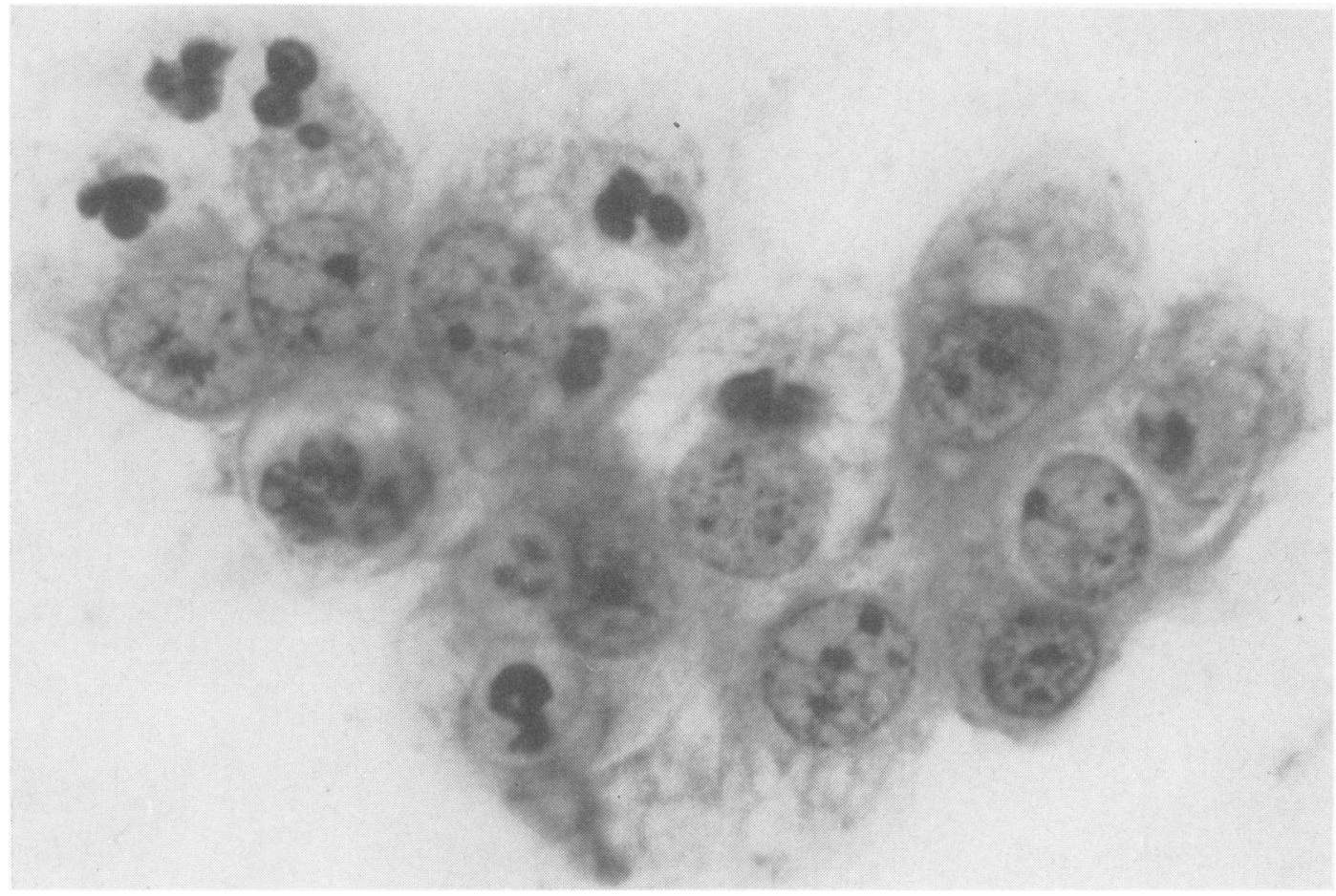

FIG. 4. A sheet of gastric epithelial cells recovered by means of lavage. Polymorphonuclear leucocytes abound but the exact relationship of most of these to the epithelial cells is not clear. Several of the leucocytes are surrounded by a clear halo suggesting that they are present as cytoplasmic inclusions. Papanicolaou $\times 840$. Enlarged $\times 4$.

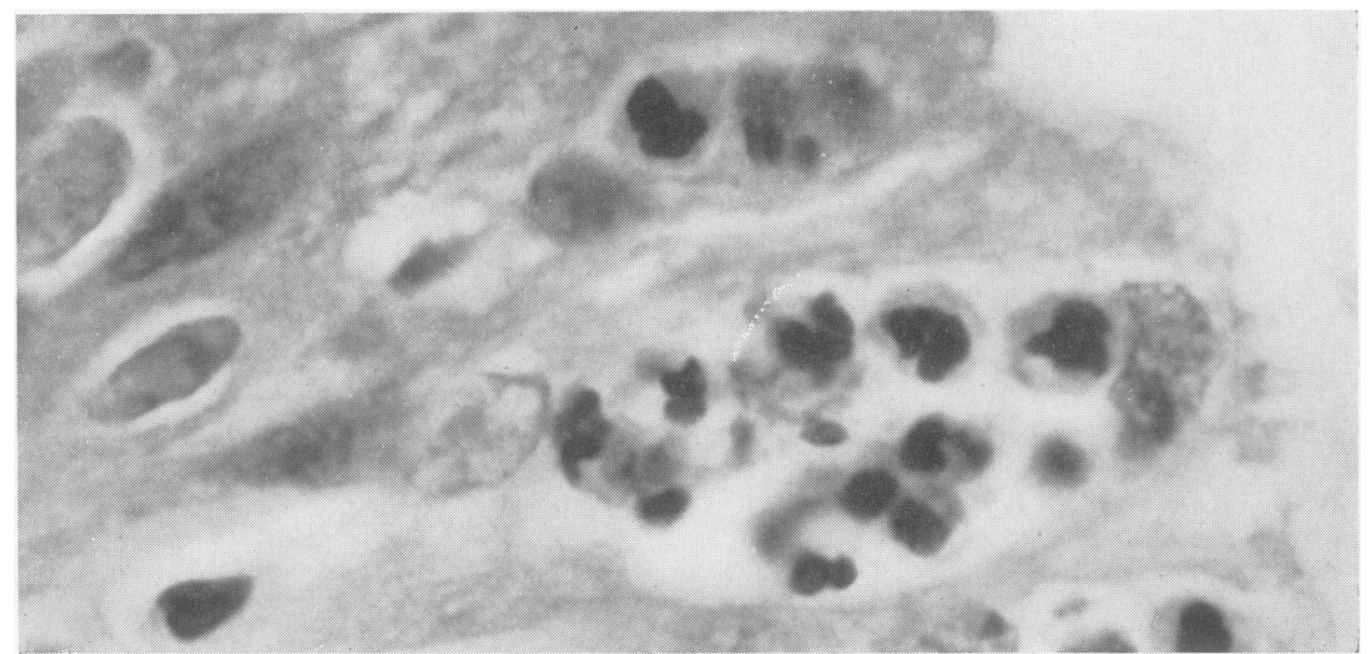

FIG. 5. Groups of leucocytes within gastric epithelum viewed in histological section. Haematoxylin and eosin $\times 840$. Enlarged $\times 4$. 


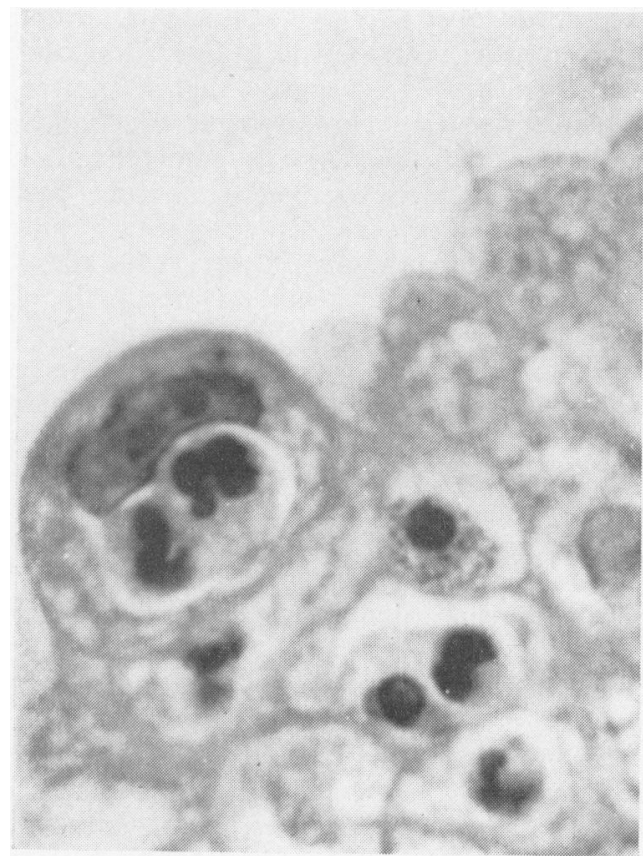

FIG. 6. Cells near the surface of gastric epithelium illustrating the histological appearances of leucocytic permeation. Haematoxylin and eosin $\times 840$. Enlarged $\times 4$.

FIG. 7. Biopsy specimen from the gastric antrum in which polymorphonuclear infiltration into the lamina propria can be seen. Polymorphs appear to be traversing the epithelium. Detachment of superficial fragments of epithelium, into which leucocytes have permeated, is occurring. Haematoxylin and eosin $\times 400$. Enlarged $\times 2$.

FIG. 6

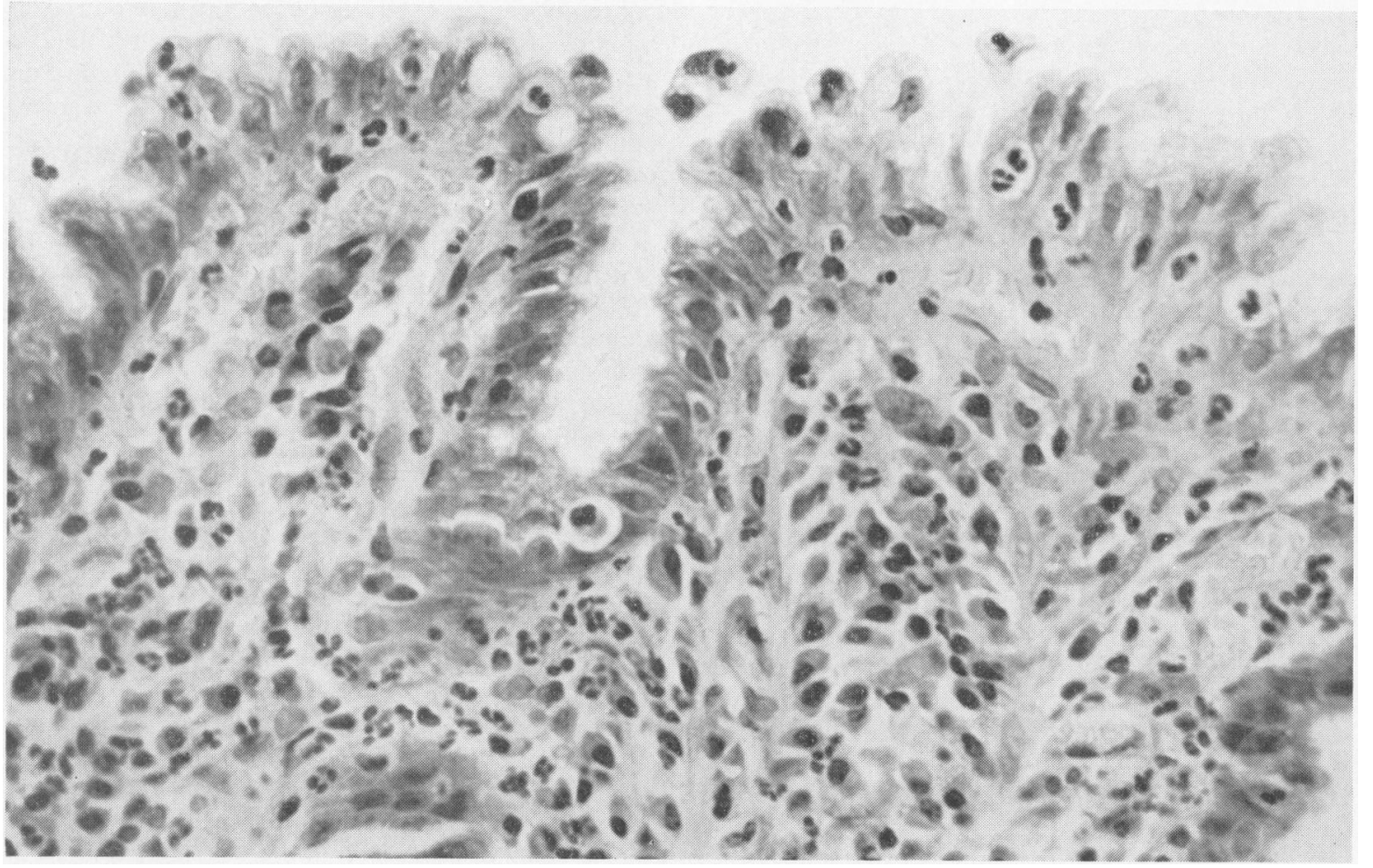

FIG. 7 
present in cytoplasm and nuclei. In some the cytoplasm had become vacuolated and foamy in appearance, the cell margin eventually becoming indistinct. Karyorrhexis was displayed in some nuclei and at times nuclear material appeared to have been extruded altogether from a cell. The cells, particularly when detached from sheets of surface epithelium, tended to lose their columnar and polyhedral shapes and become globular. In some of these epithelial cells constituents of other cells were clearly visible within the cytoplasm. Full details of these cells, which appeared to be truly intracytoplasmic, were not easily discernible, but nuclei, usually bilobed or multilobed, were most readily identified. Surrounding the intracytoplasmic leucocytes a clear halo was often visible.

Gastric biopsies from six of the patients showed changes of atrophic gastritis; in two superficial gastritis was present. In all sections leucocytes could be seen in the lining epithelium and it might be inferred from their appearance that they were passing through the epithelium either singly or in groups. The phenomenon is illustrated in Figures 5 to 7. Numerous polymorphonuclear leucocytes are seen in the superficial part of the lamina propria in Fig. 7, and an invasion of the epithelium by cells with multilobed nuclei is apparent. A clear halo surrounds most of these invading cells. On the surface of the mucosa several epithelial fragments give the appearance of becoming detached and leucocyte inclusions are visible within these.

\section{DISCUSSION}

Whereas detailed descriptions of the histological features of superficial and atrophic gastritis are available, the cytological changes that occur in the gastric epithelium have received comparatively little attention. The observations of Magnus (1946) and Motteram (1951) on leucocytes appearing to migrate through the gastric epithelium have already been mentioned. Wood and Taft (1958) commented similarly on the appearance of superficial and atrophic gastritis. In superficial gastritis 'the pathological range is considered to be reached when there is more pronounced plasma cell infiltration, and polymorphonuclear cells are seen migrating singly or in groups from the lamina propria to penetrate the superficial epithelium and lie in the pits of superficial mucus'. In atrophic gastritis the changes in the superficial portion of the mucosa are identical, but in addition there is moderate or severe atrophy of the glands with an extension of inflammatory changes to deeper layers. Plasma cells and polymorphonuclear leucocytes are seen mainly in the superficial lamina propria with lymphocytes more prevalent in deeper layers.
The present observations in which fragments of leucocytes have been noted in the cytoplasm of exfoliated epithelial cells suggest that at least some of the leucocytes have permeated into the epithelium rather than simply migrated through it. Although leucocytes are seldom seen in the normal gastric epithelium their presence is well known in the columnar epithelial lining of the small intestine. These are usually assumed to be lymphocytes. The phenomenon whereby living lymphocytes become adherent to other cells or apparently pass over and into them was termed 'emperipolesis' by Humble, Jayne, and Pulvertaft (1956), who studied the peculiar relationship between lymphocytes and malignant cells and cells in mitosis. The morphological features displayed by most of the leucocyte inclusions noted in gastric epithelium in the present study suggest that they are polymorphs; in addition the biopsy sections showed multilobed polymorphs lying in the superficial part of the lamina propria. In this context, the observations of Kingsbury (1944) made on lymphocytes which invaded epithelium covering the palatine tonsil of the cat should be mentioned. He noted that, once within epithelial cells, lymphocytes speedily lost any basophilia they might possess, the cytoplasm became scanty or indistinguishable, and the nucleus tended to enlarge and become less chromatic. Moreover the nuclei tended to become polymorphous, a feature he interpreted to mean incipient fragmentation and degeneration. From the present findings an assertion that the leucocyte inclusions in gastric epithelium were necessarily all derived from polymorphs rather than lymphocytes would not be justified.

The cytological observations now reported in a few patients with gastritis favour the occurrence of epithelial infiltration by leucocytes rather than a simple transmigration of white cells through the epithelium. The leucocytic invasion of the epithelium appears to include passage of leucocytes across the cell borders of some epithelial cells. That infiltration of this type is not a significant feature of normal gastric mucosa is supported by the findings of Palmer (1954) who took serial mucosal biopsies from a healthy adult volunteer before and after a test meal. There was no increase in the cellular content of the lamina propria, nor was there evidence of 'leucopedesis' through the epithelium, in the tissue obtained either before or after the meal.

\section{SUMMARY}

Intracytoplasmic inclusions of leucocytes were found in epithelial cells which had been exfoliated from the gastric mucosa of certain patients with superficial 
or atrophic gastritis. Gastric mucosal biopsies from the same patients showed leucocytes in the epithelium. Some clarification of the relationship of these invading leucocytes to gastric epithelial cells in gastritis has been possible.

I am indebted to Mr. John King of the Bernhard Baron Institute of Pathology, The London Hospital, for the photomicrographs. The cost of materials was met by a personal grant from the London Hospital Research fund.

\section{REFERENCES}

Crosby, W. H., and Kugler, H. W. (1957). Intraluminal biopsy of the small intestine. The intestinal biopsy capsule. Amer. J. dig. Dis., 2, 236-241.
Gibbs, D. D. (1962). Exfoliative cytology of the stomach. D.M. Thesis, Oxford.

Humble, J. G., Jayne, W. H. W., and Pulvertaft, R. J. V. (1956) Biological interaction between lymphocytes and other cells. Brit. J. Haemat., 2, 283-294.

Kingsbury, B. F. (1944). Tonsillar epithelium and the lymphocyte: an examination of the palatine tonsil of the cat. Amer.J. Anat., $75,233-261$.

Konjetzny, G. E. (1930). Die Deckepithelveränderungen der Magenschleimhaut bei akuter Gastritis. Virchows Arch. path. Anat., 275, 816-827.

Magnus, H. A. (1946). The pathology of simple gastritis. J. Path. Bact., 58, 431-439.

Motteram, R. (1951). A biopsy study of chronic gastritis and gastric atrophy. Ibid., 63, 389-394.

Palmer, E. D. (1954). Gastritis: a revaluation. Medicine (Baltimore), 33, $199-290$.

Papanicolaou, G. N. (1942). A new procedure for staining vaginal smears. Science, 95, 438-439.

Wood, I. J., and Taft, L. I. (1958). Diffuse Lesions of the Stomach. Arnold, London. 